\title{
Rethinking revascularization
}

Choosing the appropriate treatment for patients who present with chest pain is a daily dilemma. Now, a study in Mayo Clinic Proceedings has shown that chest pain unit (CPU) protocols safely identify patients at low risk of cardiac events, but that early revascularization does not improve 1-year outcomes in high-risk patients.

Alongside diagnostic tests, CPU protocols are used to assess patients and determine their cardiac risk index. Prompt revascularization is known to improve the prognosis of patients with ischemia-related chest pain; however, a number of patients are classed as high risk in the absence of any ischemic markers, and the benefit of invasive management has not been shown in this subgroup of patients. The investigators, therefore, wanted to determine whether early revascularization in these patients influenced their prognosis, and whether it was safe to discharge patients deemed to be at low risk.

Of the 1,088 patients assessed, those discharged after exercise testing (group $1 ; n=424$ ), and those in whom unstable angina was ruled out after further evaluation (group 2; $n=208$ ), were not treated with revascularization. Patients in whom unstable angina was confirmed or could not be eliminated (group 3; $n=456$ ) were either treated with revascularization (41\%) or conservatively managed. The 1-year survival rates were much lower in group 3 than in groups 1 and 2. Although group 3 patients treated with revascularization showed no improvement in 1-year outcomes compared with those who were not, early revascularization was found to decrease the need for post-discharge revascularization. A CPU protocol, therefore, needs to be flexible and allow consideration of different treatments depending on the patient's risk index.

\section{Erika Kennington}

Original article Sanchis, J. et al. Efficacy of coronary revascularization in patients with acute chest pain managed in a chest pain unit. Mayo Clin. Proc. 84, 323-329 (2009). 